\title{
Cosmic magnetic fields from velocity perturbations in the early Universe
}

\author{
Gerold Betschart $\uparrow \S$, Peter K. S. Dunsby $\dagger+$ and Mattias \\ Marklund $\S$ \\ † Department of Mathematics and Applied Mathematics, University of Cape Town, \\ 7701 Rondebosch, South Africa \\ ‡ South African Astronomical Observatory, Observatory 7925, Cape Town, South \\ Africa \\ $\S$ Department of Electromagnetics, Chalmers University of Technology, SE-412 96 \\ Göteborg, Sweden
}

\begin{abstract}
We show, using a covariant and gauge - invariant charged multifluid perturbation scheme, that velocity perturbations of the matter - dominated dust Friedmann - Lemaitre - Robertson - Walker (FLRW) model can lead to the generation of cosmic magnetic fields. Moreover, using cosmic microwave background (CMB) constraints, it is argued that these fields can reach strengths of between $10^{-28}$ and $10^{-29} \mathrm{G}$ at the time the dynamo mechanism sets in, making them plausible seed field candidates.

PACS numbers: 52.27.Ny, 04.40.-b, 98.80.-k
\end{abstract}

\section{Introduction}

Cosmological magnetic fields have generated considerable debate ever since they were first observed [1]. Such large scale fields occur in galaxy clusters, high redshift condensations, spiral and disc galaxies, and have strengths between $10^{-7}$ to $10^{-5} \mathrm{G}[2]$. While the structure of the magnetic fields in spiral galaxies appear to indicate that they were generated and sustained by a dynamo mechanism [3], the origin of the necessary seed field is still unknown. For the dynamo mechanism to be successful, the present day strength of these seed fields must lie within a range of $10^{-23}$ [4] to $10^{-30} \mathrm{G}$ [5], the lower value being for a dark energy dominated spatially flat universe.

Primordial magnetic fields are of particular interest since they explain both the fields seen in nearby galaxies as well as those detected in galaxy clusters and highly redshifted condensations. Several different schemes generating these fields have been suggested, many of them based on breaking the conformal invariance of electromagnetism. Since this can be achieved in more than one way, this explains the variety of the proposed mechanisms in the literature, such as coupling the photon to a scalar field either during inflation or in the subsequent era of preheating [6] (see [7] for a critique), or assuming the breakdown of Lorentz invariance either in the context 
of string theory and non - commutative varying speed of light theories, or due to the dynamics of large extra dimensions [8]. Although these proposals successfully explain the generation of primordial fields, it is often done at the expense of simplicity because they extend the physical parameter space.

Recently it has been suggested that if a magnetic seed field exists below the necessary strength for the dynamo mechanism to work, gravitational waves could be used to pump the magnetic field by many orders of magnitude [9]. The mechanism uses the primordial gravitational wave spectrum generated during inflation, and couples this via Maxwell's equations to the magnetic seed field. This model has the attractive feature that one need not introduce new physics or extend the number of fields considered. Of course, a disadvantage is that it relies on the yet unobserved inflationary, model dependent gravitational wave spectrum, and that still requires another model to create the weak seed field.

With this in mind, we propose a mechanism based solely on the physics of self gravitating plasmas. Since velocity and density perturbations naturally occur in the early Universe, it is interesting to examine whether such perturbations can induce magnetic fields which can be sustained at appreciable levels until the onset of non - linear gravitational collapse (for a discussion of density perturbations in the presence of weak magnetic fields see, e.g., [10-12]. The mechanism is similar to Harrison's protogalaxy model [13], and the Biermann battery effect [14], in the sense of yielding vorticity driven magnetic fields, but we note that the battery effect in our formalism would be of second order, while the Harrison effect relies on Thomson scattering.

Using a recently developed formalism for describing perturbations in a general relativistic multi - component plasma [15], it is found that the value of the magnetic field generated by primordial velocity perturbations is well within the limits required if the dynamo mechanism is to work. The limitations of the method, as well as the results, are then discussed.

\section{Preliminaries}

To begin with, we present some of the key equations from [15] which were obtained by linearizing the exact Einstein - Maxwell equations using a two - parameter approximation scheme characterized by

- $\varepsilon_{g}$ - gravitational: $\sigma_{a b}, \omega_{a b}, \tilde{\nabla} \sigma_{a b}, \tilde{\nabla} \omega_{a b}$, etc.

- $\varepsilon_{e m}$ - electromagnetic: $E_{a}, B_{a}$.

In this way, terms which are second order in the gravitational variables, $\varepsilon_{g}^{2}$, second order in the induced electromagnetic fields, $\varepsilon_{e m}^{2}$ and all cross terms $\varepsilon_{g} \varepsilon_{e m}$ are neglected.

\subsection{First Order Multifluid equations}

Using the above approximation scheme, the Einstein - Maxwell equations are linearized around a pressure - free FLRW model. Hence all spatial gradients and velocity 
components perpendicular to the fundamental observer with 4 -velocity $u^{a}$ must vanish in the background. This implies that inhomogeneities and velocity perturbations play a first order role. In addition, the isotropy and homogeneity of the background demands that the electromagnetic field and accordingly the total charge density $\rho$ vanishes in the background.

From Maxwell's equations, we find the first order equations

$$
\begin{aligned}
& \dot{E}^{\langle a\rangle}-\operatorname{curl} B^{a}=-\epsilon_{0}^{-1} j^{\langle a\rangle}-\frac{2}{3} \Theta E^{a}, \\
& \dot{B}^{\langle a\rangle}+\operatorname{curl} E^{a}=-\frac{2}{3} \Theta B^{a}, \\
& \tilde{\nabla}_{a} E^{a}=\epsilon_{0}^{-1} \rho, \\
& \tilde{\nabla}_{a} B^{a}=0
\end{aligned}
$$

where $\operatorname{curl} B^{a}=\epsilon^{a b c} \tilde{\nabla}_{b} B_{c}$ and $\Theta$ is the cosmological expansion defined through the scale factor $S: \Theta=3 \dot{S} / S$

Neglecting all but electromagnetic interactions between the fluids (e.g. collisions), then, for each fluid species (i), conservation of energy, momentum and particle density leads to the following equations:

$$
\begin{aligned}
& \dot{\mu}_{(i)}+\mu_{(i)} \tilde{\nabla}_{a} v_{(i)}^{a}=-\mu_{(i)} \Theta, \\
& \mu_{(i)} \dot{v}_{(i)}^{\langle a\rangle}=\rho_{(i)} E^{a}-\frac{1}{3} \mu_{(i)} \Theta v_{(i)}^{a}, \\
& \dot{n}_{(i)}+n_{(i)} \tilde{\nabla}_{a} v_{(i)}^{a}=-\Theta n_{(i)} .
\end{aligned}
$$

Since we are working in the energy frame, where the total heat flux vanishes, the equation for total energy conservation is simply given by

$$
\dot{\mu}=-\Theta \mu \text {. }
$$

Because no thermal effects are included, we may express the fluids' energy density as $\mu_{i}=m_{i} n_{i}$ and the energy frame choice further implies $\sum_{i} \mu_{i} v_{i}^{a}=0$.

Specializing to two components, it is convenient to introduce the following variables

$$
\begin{aligned}
& N=n_{1}+n_{2}, \\
& n=n_{1}-n_{2}, \\
& V^{a}=\frac{1}{2}\left(v_{1}^{a}+v_{2}^{a}\right), \\
& v^{a}=\frac{1}{2}\left(v_{1}^{a}-v_{2}^{a}\right) .
\end{aligned}
$$

The following system of first order equations then follows, using equations (6) and (7):

$$
\begin{aligned}
\dot{n} & =-N \tilde{\nabla}_{a} v^{a}-\Theta n, \\
\dot{N} & =N \frac{\Delta \mu}{\mu} \tilde{\nabla}_{a} v^{a}-\Theta N, \\
\dot{v}^{\langle a\rangle} & =-\frac{e}{2}\left(\frac{1}{m_{1}}+\frac{1}{m_{2}}\right) E^{a}-\frac{1}{3} \Theta v^{a},
\end{aligned}
$$

where we defined $\Delta \mu=\mu_{1}-\mu_{2}$ and the total energy density $\mu=\mu_{1}+\mu_{2}$, respectively. Since we have $\mu_{i}=m_{i} n_{i}$, the latter can be written as

$$
\mu=\frac{1}{2}\left(m_{1}+m_{2}\right) N+\frac{1}{2}\left(m_{2}-m_{1}\right) n
$$


a relation which will be used very often in the following analysis. The Raychaudhuri equation then becomes

$$
\dot{\Theta}=-\frac{1}{3} \Theta^{2}-\frac{1}{4}\left(m_{1}+m_{2}\right) N-\frac{1}{4}\left(m_{1}-m_{2}\right) n .
$$

It is useful to introduce the following quantities, namely

$$
\begin{aligned}
Y & =\frac{n}{N}, \\
\alpha^{2} & =\frac{4 e^{2}}{3 \varepsilon_{0} m_{1} m_{2}}, \\
\beta^{2} & =\frac{e}{\varepsilon_{0}\left(m_{1}+m_{2}\right)} .
\end{aligned}
$$

The fraction $Y=n / N$ obeys a propagation equation,

$$
\ddot{Y}+\frac{2}{3} \Theta \dot{Y}+\frac{3}{4} \alpha^{2} \mu Y=0,
$$

which may be derived from the above definitions and equations (13)-(17).

\section{Velocity Induced Electromagnetic Fields}

For a cold plasma, the currents for each fluid species may be written as

$$
j_{(i)}^{a}=q_{(i)} n_{(i)} V_{(i)}^{a}=q_{(i)} n_{(i)}\left(u^{a}+v_{(i)}^{a}\right),
$$

where $q_{(i)}$ is the charge and $v_{(i)}^{a}$ is the velocity of the species under consideration. Since we require the plasma to be neutral on the whole, the species are of opposite charge. Hence, the total current $j^{a}$ appearing in Maxwell's equations reads to first order

$$
j^{a}=j_{1}^{a}+j_{2}^{a}=-e N v^{a} .
$$

From Maxwell's equations (1) -(4), using (23) and (15), one can then deduce second order wave equations for the induced electromagnetic fields. They are

$$
\begin{aligned}
& \ddot{E}_{\langle a\rangle}-\tilde{\nabla}^{2} E_{a}+\frac{5}{3} \Theta \dot{E}_{\langle a\rangle}+\left[\frac{2}{9} \Theta^{2}+\left(\frac{3}{4} \alpha^{2}+\frac{1}{3}\right) \mu\right] E_{a}=2 \beta^{2} \mu\left(\tilde{\nabla}_{a} Y-\frac{1}{3} \Theta v_{a}\right), \\
& \ddot{B}_{\langle a\rangle}-\tilde{\nabla}^{2} B_{a}+\frac{5}{3} \Theta \dot{B}_{\langle a\rangle}+\left[\frac{2}{9} \Theta^{2}+\frac{1}{3} \mu\right] B_{a}=-2 \beta^{2} \mu \operatorname{curl} v_{a} .
\end{aligned}
$$

Observe that $B_{a}$ and curl $v_{a}$ are both purely solenoidal, whereas $\tilde{\nabla}_{a} Y$ has no solenoidal part. It is worthwhile to note that the magnetic field is solely sourced by inhomogeneities in the velocity in contrast to the electric field which is sourced by inhomogeneities in the number density and velocity perturbations. Both equations look strikingly similar, the differences originating either from the total current or from a gradient in the charge density (in the case of $\tilde{\nabla}_{a} Y$ ). The additional $3 / 4 \alpha^{2}$-term in the electric wave equation comes from the non-stationarity of the total current and its largeness $-\alpha^{2} \sim 10^{42}$ for an $e^{+} e^{-}$- plasma - leads directly to the high-frequency behaviour of plasma effects, as will be shown below (see also [15] for a discussion of the high-frequency plasma mode in the gravitational instability picture).

It will be useful to introduce expansion normalized variables,

$$
\mathscr{E}_{a} \equiv \frac{E_{a}}{\Theta}, \quad \mathscr{B}_{a} \equiv \frac{B_{a}}{\Theta}, \quad \mathscr{K}_{a} \equiv \frac{\operatorname{curl} v_{a}}{\Theta} .
$$


Equations (24) and (25), together with equations for the driving terms, then read

$$
\begin{aligned}
& \ddot{\mathscr{E}}_{\langle a\rangle}-\tilde{\nabla}^{2} \mathscr{E}_{a}+\left(\Theta-\frac{\mu}{\Theta}\right) \dot{\mathscr{E}}_{\langle a\rangle}-\left[\frac{1}{9} \Theta^{2}-\left(\frac{3}{4} \alpha^{2}+\frac{1}{3}\right) \mu\right] \mathscr{E}_{a}=2 \beta^{2} \frac{\mu}{\Theta}\left(\tilde{\nabla}_{a} Y-\frac{1}{3} \Theta v_{a}\right) \\
& \dot{v}_{\langle a\rangle}+\frac{1}{3} \Theta v_{a}=-\frac{3}{8} \frac{\alpha^{2}}{\beta^{2}} \Theta \mathscr{E}_{a}, \\
& \ddot{\mathscr{B}}_{\langle a\rangle}-\tilde{\nabla}^{2} \mathscr{B}_{a}+\left(\Theta-\frac{\mu}{\Theta}\right) \dot{\mathscr{B}}_{\langle a\rangle}-\left(\frac{1}{9} \Theta^{2}-\frac{1}{3} \mu\right) \mathscr{B}_{a}=-2 \beta^{2} \mu \mathscr{K}_{a}, \\
& \dot{\mathscr{K}}_{\langle a\rangle}+\left(\frac{1}{3} \Theta-\frac{1}{2} \frac{\mu}{\Theta}\right) \mathscr{K}_{a}=\frac{3}{8} \frac{\alpha^{2}}{\beta^{2}}\left[\dot{\mathscr{B}}_{\langle a\rangle}+\left(\frac{1}{3} \Theta-\frac{1}{2} \frac{\mu}{\Theta}\right) \mathscr{B}_{a}\right] .
\end{aligned}
$$

Equation (30) follows from (26) using (28) and Maxwell's equation (2). In order to find solutions to the above equations, we extract from them the scalar and solenoidal (vector) parts (cf. Appendix B) and solve them separately.

\subsection{Scalar modes}

In analogy with (B.3), we set $V \equiv S \tilde{\nabla}^{a} v_{a}$ and $\mathscr{E} \equiv S \tilde{\nabla}^{a} \mathscr{E}_{a}$. Equation (28) then transforms into

$$
\dot{V}+\frac{1}{3} \Theta V=-\frac{3}{8} \frac{\alpha^{2}}{\beta^{2}} \Theta \mathscr{E}=\frac{3}{4} \alpha^{2} \mu S Y,
$$

where the last equality is a direct consequence of Maxwell's equation (3). Combining $\dot{Y}=-V / S$ with (31) and using (8) and (17) together with

$$
S \tilde{\nabla}^{a} \tilde{\nabla}^{2} \mathscr{E}_{a}=\tilde{\nabla}^{2} \mathscr{E}+\left(-\frac{2}{9} \Theta^{2}+\frac{2}{3} \mu\right) \mathscr{E}
$$

one can show that the scalar part of the electric wave equation (27) reduces to

$$
\ddot{\mathscr{E}}+\left(\frac{4}{3} \Theta-\frac{\mu}{\Theta}\right) \dot{\mathscr{E}}+\left[\frac{2}{9} \Theta^{2}+\left(\frac{3}{4} \alpha^{2}-\frac{1}{2}\right) \mu\right] \mathscr{E}=0 .
$$

It is also easy to see that equation (31) additionally gives rise to propagation equations for $V$ and $Y$ :

$$
\begin{aligned}
& \ddot{V}+\frac{1}{3} \Theta \dot{V}+\left[-\frac{1}{9} \Theta^{2}+\left(\frac{3}{4} \alpha^{2}-\frac{1}{6}\right) \mu\right] V=0, \\
& \ddot{Y}+\frac{2}{3} \Theta \dot{Y}+\frac{3}{4} \alpha^{2} \mu Y=0 .
\end{aligned}
$$

Hence, equations (33)-(35) all emanate from (31). We now specialise our considerations to a flat FLRW background with zero cosmological constant, for which $\mu=1 / 3 \Theta^{2}$ and $\Theta=2 / t$ always hold, solutions may easily be obtained:

$$
\begin{aligned}
& V(\tau)=\frac{1}{\sqrt{\tau}}\left\{V_{i} \cos (\omega \ln \tau)+\frac{1}{\omega}\left(\frac{1}{2} V_{i}+V_{i}^{\prime}\right) \sin (\omega \ln \tau)\right\} \\
& \mathscr{E}(\tau)=-\frac{4}{9} \frac{\beta^{2}}{\alpha^{2}} \frac{1}{\sqrt{\tau}}\left\{\left(2 V_{i}+3 V_{i}^{\prime}\right) \cos (\omega \ln \tau)+\frac{\left(2-18 \alpha^{2}\right) V_{i}+3 V_{i}^{\prime}}{6 \omega} \sin (\omega \ln \tau)\right\}, \\
& Y(\tau)=\frac{t_{i}}{3 S_{i}} \frac{1}{\alpha^{2}} \frac{1}{\tau^{1 / 6}}\left\{\left(2 V_{i}+3 V_{i}^{\prime}\right) \cos (\omega \ln \tau)+\frac{\left(2-18 \alpha^{2}\right) V_{i}+3 V_{i}^{\prime}}{6 \omega} \sin (\omega \ln \tau)\right\} .
\end{aligned}
$$


Here, we introduced the dimensionless time-coordinate $\tau \equiv t / t_{i}$, where $t_{i}$ denotes some arbitrary initial time. The scale factor becomes now $S(\tau)=S_{i} \tau^{2 / 3}$. Initial conditions of the velocity perturbation are chosen to be $V_{i} \equiv V(1)$ and $V_{i}^{\prime} \equiv V^{\prime}(1)$ (a prime stands for $\left.\partial_{\tau}\right)$. The frequency of the solutions is proportional to $\omega \equiv \sqrt{\alpha^{2}-1 / 36}$ and grows logarithmically in time. The solutions show the same high - frequency behaviour that was obtained in [15].

\subsection{Vector modes}

According to (B.6), we set $\tilde{\mathscr{E}}_{a} \equiv S \operatorname{curl} \mathscr{E}_{a}$ etc., and obtain from equations (27)-(30)

$\ddot{\tilde{\mathscr{E}}}_{\langle a\rangle}-\tilde{\nabla}^{2} \tilde{\mathscr{E}}_{a}+\left(\Theta-\frac{\mu}{\Theta}\right) \dot{\tilde{\mathscr{E}}}_{\langle a\rangle}+\left[-\frac{1}{9} \Theta^{2}+\left(\frac{3}{4} \alpha^{2}+\frac{1}{3}\right) \mu\right] \tilde{\mathscr{E}}_{a}=-\frac{2}{3} \beta^{2} \mu \tilde{v}_{a}$

$\dot{\tilde{v}}_{\langle a\rangle}+\frac{1}{3} \Theta \tilde{v}_{a}=-\frac{3}{8} \frac{\alpha^{2}}{\beta^{2}} \Theta \tilde{\mathscr{E}}_{a}$,

$\ddot{\mathscr{\mathscr { B }}}_{\langle a\rangle}-\tilde{\nabla}^{2} \tilde{\mathscr{B}}_{a}+\left(\Theta-\frac{\mu}{\Theta}\right) \dot{\mathscr{\mathscr { B }}}_{\langle a\rangle}+\left(-\frac{1}{9} \Theta^{2}+\frac{1}{3} \mu\right) \tilde{\mathscr{B}}_{a}=-2 \beta^{2} \mu \tilde{\mathscr{K}}_{a}$,

$\dot{\mathscr{K}}_{\langle a\rangle}+\left(\frac{1}{3} \Theta-\frac{1}{2} \frac{\mu}{\Theta}\right) \tilde{\mathscr{K}}_{a}=\frac{3}{8} \frac{\alpha^{2}}{\beta^{2}}\left[\dot{\mathscr{\mathscr { B }}}_{\langle a\rangle}+\left(\frac{1}{3} \Theta-\frac{1}{2} \frac{\mu}{\Theta}\right) \tilde{\mathscr{B}}_{a}\right]$.

Specialising to a flat FLRW background and performing a harmonic decomposition (see Appendix B), these equations become

$$
\begin{aligned}
& \mathscr{E}^{\prime \prime}+\frac{4}{3 \tau} \mathscr{E}^{\prime}+\left[\frac{L^{2}}{\tau^{4 / 3}}+\frac{\alpha^{2}}{\tau^{2}}\right] \mathscr{E}=-\frac{8 \beta^{2}}{9 \tau^{2}} v, \\
& v^{\prime}+\frac{2}{3 \tau} v=-\frac{3 \alpha^{2}}{4 \beta^{2} \tau} \mathscr{E}, \\
& \mathscr{B}^{\prime \prime}+\frac{4}{3 \tau} \mathscr{B}^{\prime}+\frac{L^{2}}{\tau^{4 / 3}} \mathscr{B}=-\frac{4 \beta^{2}}{3 \tau^{2}} \mathscr{K}, \\
& \mathscr{K}^{\prime}+\frac{1}{3 \tau} \mathscr{K}=\frac{3 \alpha^{2}}{8 \beta^{2}}\left[\mathscr{B}^{\prime}+\frac{1}{3 \tau} \mathscr{B}\right],
\end{aligned}
$$

where we have dropped the index $V$ [denoting that the variables in equations (43)-(46) are vector harmonic coefficients], and we have defined $\tau \equiv t / t_{i}$ and used

$$
\left(\frac{k t_{i}}{S}\right)^{2}=\left(\frac{4 \pi}{3}\right)^{2}\left(\frac{\lambda_{H}}{\lambda}\right)_{i}^{2} \frac{1}{\tau^{4 / 3}}=L^{2} \frac{1}{\tau^{4 / 3}}
$$

for the contribution of the Laplacian in (39) and (41), respectively. In (47), $\lambda_{H}=1 / H$ is the Hubble length, $\lambda$ is the physical wavelength associated with the comoving wavenumber $k=2 \pi S / \lambda$ and the index i stands for evaluation at initial time $t_{i}$. If the wavelength of the mode is much greater than the horizon, eg. $\lambda_{i} \gg \lambda_{H_{i}}$, we may neglect the terms containing $L^{2}$. However, we can neglect that term in (43) and the system (45) - (46) throughout, because the $\alpha^{2}$ - term dominates as long as $\tau L^{3} \ll \alpha^{3}$ holds and this criterion is only violated for very late times or ultra - short wavelengths. It follows that the above equations can then be solved analytically and the general (real) 
solutions are found to be

$v(\tau)=C_{1}+\frac{1}{\sqrt{\tau}}\left\{C_{2} \cos (\omega \ln \tau)+C_{3} \sin (\omega \ln \tau)\right\}$,

$\mathscr{E}(\tau)=-\frac{8}{9} \frac{\beta^{2}}{\alpha^{2}} C_{1}-\frac{2}{9} \frac{\beta^{2}}{\alpha^{2}} \frac{1}{\sqrt{\tau}}\left\{\left(C_{2}+6 \omega C_{3}\right) \cos (\omega \ln \tau)+\left(C_{3}-6 \omega C_{2}\right) \sin (\omega \ln \tau)\right\}$,

$\mathscr{K}(\tau)=\frac{1}{\tau^{1 / 6}}\left\{D_{1} \cos (\tilde{\omega} \ln \tau)+D_{2} \sin (\tilde{\omega} \ln \tau)\right\}$,

$\mathscr{B}(\tau)=D_{3} \frac{1}{\tau^{1 / 3}}+\frac{8}{3} \frac{\beta^{2}}{\alpha^{2}} \frac{1}{\tau^{1 / 6}}\left\{D_{1} \cos (\tilde{\omega} \ln \tau)+D_{2} \sin (\tilde{\omega} \ln \tau)\right\}$,

where $\omega=\sqrt{\alpha^{2}-1 / 36}$ and $\tilde{\omega}=\sqrt{\alpha^{2} / 2-1 / 36}$. Since we think of the electromagnetic fields as being generated by the velocity perturbations, we choose initial conditions for $v$ and $\mathscr{K}$, respectively: namely, $v_{i} \equiv v(1), v_{i}^{\prime} \equiv v^{\prime}(1)$ and $\mathscr{K}_{i} \equiv \mathscr{K}(1), \mathscr{K}_{i}^{\prime} \equiv \mathscr{K}^{\prime}(1)$. Notice that the system of equations (43) and (44) is equivalent to a third order ODE for $v$ (or $\mathscr{E}$ ), for any value of $L$, while the system of equations (45) and (46) is equivalent to a second order ODE for $\mathscr{K}$, for $L=0$ only. However, the vector modes of $\mathscr{K}_{a}$ are linearly related to those of $v_{a}$ [according to (26)], thus we have $S \Theta \mathscr{K} \sim v$. Therefore consistency requires the vanishing of the integration constant $C_{1}$. For this set of initial conditions, the integration constants $C_{i}$ and $D_{i}$ become

$$
C_{2}=v_{i}, \quad C_{3}=\frac{v_{i}+2 v_{i}^{\prime}}{2 \omega}, \quad D_{1}=\mathscr{K}_{i}, \quad D_{2}=\frac{\mathscr{K}_{i}+6 \mathscr{K}_{i}^{\prime}}{6 \tilde{\omega}},
$$

while there is no restriction for $D_{3}$, since we put $L \simeq 0$, but with the assumption that the initial magnetic field vanishes, we get a relation between $D_{3}$ and $\mathscr{K}_{i}$. The solutions for the velocity perturbation and the (expansion normalized) electric field agree then with those found in the scalar case and show the same behaviour in time, as expected. The induced expansion normalized magnetic field attains two parts, a standard decaying part and a weakly decaying oscillatory part due to the plasma.

\section{Applications}

\subsection{Generated fields}

Observe that the magnetic field (51) is rather slowly decaying and therefore still could play a role in some astrophysical processes under favourable conditions. If we assume that the velocity induced magnetic field vanishes initially, then we may approximate its magnitude by the following expression:

$$
|\mathscr{B}(\tau)| \lesssim \frac{8}{3} \frac{\beta^{2}}{\alpha^{2}} \frac{1}{\tau^{1 / 6}}\left(1-\frac{1}{\tau^{1 / 6}}\right) \mathscr{K}_{i},
$$

where $\mathscr{K}_{i}$ is the magnitude of the initial velocity curl perturbation. Restoring SI units, we find for the physical magnetic field

$|B(t)| \lesssim\left(\frac{m_{1}}{m_{e}}\right)\left(\frac{m_{1}}{m_{e}}\right)\left(\frac{m_{e}}{m_{1}+m_{2}}\right) \mathscr{K}_{i}\left(\frac{t_{i}}{t}\right)^{1 / 6}\left[1-\left(\frac{t_{i}}{t}\right)^{1 / 6}\right] \frac{1}{t} \times 2 \times 10^{-7} \mathrm{G}$, 


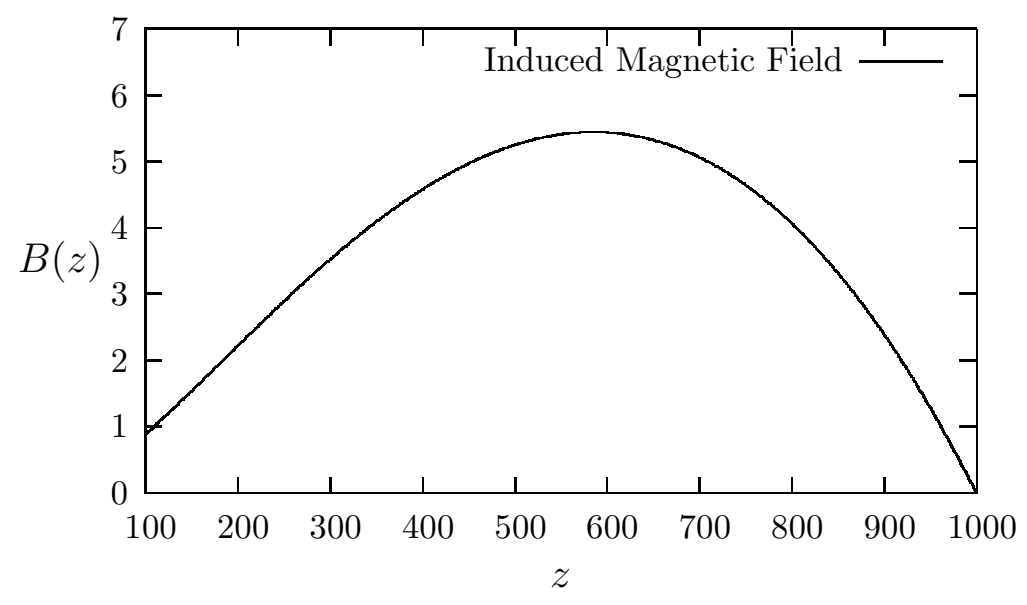

Figure 1. Generated magnetic field in units of $10^{-27} \mathrm{G}$, for the initial and final redshift 1000 and 100 , respectively.

or alternatively, using $1+z=\left(1+z_{i}\right) / \tau^{2 / 3}$,

$$
|B(z)| \lesssim \mathscr{K}_{i} h\left(\frac{1+z}{1+z_{i}}\right)^{1 / 4}\left[1-\left(\frac{1+z}{1+z_{i}}\right)^{1 / 4}\right](1+z)^{3 / 2} \times 10^{-24} \mathrm{G},
$$

where we also have employed $\Theta=3 H=3 H_{0}(1+z)^{3 / 2}$ with $H_{0}=h(9.8 \mathrm{Gyrs})^{-1}$, and neglected the mass factor. $\neq$ Thus, velocity curl perturbations of magnitude $\mathscr{K}_{i} \sim 10^{-5}$ in an $e^{+} e^{-}$- plasma starting after decoupling, when Thomson scattering becomes negligible $(z \sim 1000)$, would induce a magnetic field with a strength between $10^{-26}$ and $10^{-27} \mathrm{G}$ at a redshift of $z \sim 100$ (see figure 1 above), a redshift well within the limits before nonlinear effects become important. Redshifting to $z \simeq 10$, at the onset of the dynamo mechanism [1] reduces the field strength to between $10^{-28}$ and $10^{-29} \mathrm{G} . \S$

The above argument is also applicable for the decaying electric field (for both scalar and vector modes). If we require the velocity induced electric field to vanish initially, then its envelope is given by

$$
\left|\mathscr{E}_{\text {env }}(\tau)\right|=\frac{4}{3} \frac{\beta^{2}}{\alpha} v_{i} \frac{1}{\sqrt{\tau}},
$$

where $v_{i}$ is the magnitude of the initial scalar velocity perturbation. We resort once again to SI units and find for the physical field

$\left|E_{\text {env }}(t)\right|=\left(\frac{m_{1}}{m_{e}}\right)\left(\frac{m_{2}}{m_{e}}\right)\left(\frac{m_{e}}{m_{1}+m_{2}}\right) v_{i}\left(\frac{t_{i}}{t}\right)^{1 / 2} \frac{1}{t} \times 2 \times 10^{-3} \mathrm{Vm}^{-1}$,

or alternatively,

$$
\left|E_{\text {env }}(z)\right|=v_{i} h \frac{(1+z)^{9 / 4}}{\left(1+z_{i}\right)^{3 / 4}} \times 2 \times 10^{-23} \mathrm{Vm}^{-1} .
$$

$\ddagger$ The mass parameter in (55) and (57) is always of the order of one for an electron-positron- or an electron-ion-plasma.

$\S$ The constraint concerning the initial velocity perturbation $\mathscr{K}_{i}$ and $v_{i}$, respectively, stems from the standard CMB results [16]. 
Hence, using the same data as above, i.e., velocity perturbations of magnitude $v_{i} \sim 10^{-5}$ in an $e^{+} e^{-}$-plasma starting after decoupling $(z \sim 1000)$, would lead to an induced electric field of strength $\sim 10^{-26} \mathrm{Vm}^{-1}$ at $z=100$.

Comparing the energy density of the induced electric and magnetic fields, we

find that $E_{\text {env }}^{2} / c^{2} B^{2} \sim 10^{-15}$, thus showing the well known fact that the electric field contribution, due to Debye shielding, is negligible in a cosmological context.

\section{Summary and discussion}

In this paper, we have considered the induced electromagnetic fields due to velocity perturbations in a charged multifluid, using a gauge - invariant, covariant approach. We have, in a self - consistent manner, investigated the behaviour of the interacting fluids and electromagnetic fields in the case of a flat FLRW background. In the matter dominated era, due to the non - vanishing vorticity of the velocity perturbations in the two - component fluid, we find that there is a net magnetic field with a magnitude between $10^{-30}$ and $10^{-31} \mathrm{G}$ today. Since velocity perturbations are naturally occurring in the early Universe, this field thus represents a suitable candidate for a seed field, which could be amplified by the galactic dynamo. Moreover, the model is self - consistent, and does not invoke any other physics than general relativity and classical electrodynamics

The notion of cosmic magnetic fields is nowadays generally accepted, and observations seems to indicate their presence on all scales of the Universe. On the other hand, the existing standard models for the generation of these fields [1] all require a seed field, and it is still somewhat of a mystery as to where this primordial field stems from. There have through the years been a number of suggestions for the origin of the seeds, many of them making use of yet to be confirmed physics. At the same time, CMB data is getting more and more detailed [17], and we now have a very good handle on the size of the different types of perturbation that occur in the early Universe. Since it is highly plausible that the plasma state is a good approximation of the cosmological fluid at certain stages of the evolution of the Universe, the model presented here lends itself naturally to the analysis of a possible source of the much sought-after magnetic seed field. In many of the classical mechanisms for generating the galactic and extragalactic magnetic fields, the vorticity of the fluid plays a crucial role in the generation of the magnetic fields $[13,14]$. This, in conjunction with the aforementioned CMB data, gives our model the attractive feature of being not only internally consistent, but also consistent with present day cosmological data. On the other hand, scattering and thermal effects have been neglected, and would, if included, surely contribute to the dynamics of the fluids in a nontrivial and important way, but these issues are left for future studies. We note however that including a radiation gas and Thomson scattering would bring the model to a form more accurately describing the plasma at pertinent epochs, as well as bringing it closer to Harrison's protogalaxy model. 
Cosmic magnetic fields from velocity perturbations in the early Universe

\section{Acknowledgments}

We like to thank Christos Tsagas for helpful discussions and comments. This research was supported by NRF (South Africa) and Sida (Sweden).

\section{Appendix A. Some commutator expressions}

In this section, we give some useful expressions for commuting spatial derivatives, up to first order, in the case of dust spacetimes (see also [18]). We will assume in the following that $\tilde{\nabla} N$ and $X_{a}$ are first order quantities.

$$
\begin{aligned}
& \left(\tilde{\nabla}^{2} N\right)=\tilde{\nabla}^{2} \dot{N}-\frac{2}{3} \Theta \tilde{\nabla}^{2} N, \\
& \tilde{\nabla}_{[a} \tilde{\nabla}_{b]} \tilde{\nabla}_{c} N=\left(\frac{1}{9} \Theta^{2}-\frac{1}{3} \mu\right) \tilde{\nabla}_{[a} N h_{b] c}, \\
& \tilde{\nabla}_{a} \tilde{\nabla}^{2} N=\tilde{\nabla}^{2} \tilde{\nabla}_{a} N+\left(\frac{2}{9} \Theta^{2}-\frac{2}{3} \mu\right) \tilde{\nabla}_{a} N, \\
& \tilde{\nabla}_{[a} \tilde{\nabla}_{b]} \tilde{\nabla}^{c} X^{d}=\left(-\frac{1}{9} \Theta^{2}+\frac{1}{3} \mu\right)\left[h_{[a}^{c} \tilde{\nabla}_{b]} X^{d}+h_{[a}^{d} \tilde{\nabla}^{c} X_{b]}\right], \\
& \tilde{\nabla}_{a} \tilde{\nabla}^{2} X_{b}=\tilde{\nabla}^{2} \tilde{\nabla}_{a} X_{b}+\left(\frac{2}{9} \Theta^{2}-\frac{2}{3} \mu\right)\left[\tilde{\nabla}_{a} X_{b}+\tilde{\nabla}_{b} X_{a}-h_{a b} \tilde{\nabla}_{c} X^{c}\right], \\
& S \varepsilon^{a b c} \tilde{\nabla}_{b} \tilde{\nabla}^{2} X_{c}=\tilde{\nabla}^{2}\left(S \varepsilon^{a b c} \tilde{\nabla}_{b} X_{c}\right) .
\end{aligned}
$$

\section{Appendix B. Isolating scalar and vector modes}

We define our harmonics as eigenfunctions of the Laplace-Beltrami operator $[19,20]$

$$
\tilde{\nabla}^{2} Q=-\frac{k^{2}}{S^{2}} Q, \quad \dot{Q}=0,
$$

where $\mathrm{Q}$ stands for a scalar, vector or tensor harmonic. For example, a first order vector field $X^{a}$ may be expanded covariantly in scalar and vector harmonics

$$
X^{a}=X_{S} Q_{S}^{a}+X_{V} Q_{V}^{a},
$$

where an implicit summation in this expansion is understood. In order to extract the

purely scalar modes, $X_{S}$, of a first order vector field, one basically takes the divergence (multiplied by the scale factor $S$ for convenience) and readily obtains the following relations:

$$
\begin{aligned}
X & \equiv S \tilde{\nabla}_{b} X^{b}=X_{S}\left(k Q_{S}\right), \\
\dot{X} & =S \tilde{\nabla}_{b} \dot{X}^{b}=\dot{X}_{S}\left(k Q_{S}\right), \\
\ddot{X} & =S \tilde{\nabla}_{b} \ddot{X}^{b}=\ddot{X}_{S}\left(k Q_{S}\right) .
\end{aligned}
$$

The solenoidal modes, $X_{V}$, can be obtained by applying curl and noting that the curly harmonics $\tilde{Q}_{V}^{a} \equiv S \epsilon^{a b c} \tilde{\nabla}_{b} Q_{c}^{V}$ also satisfy relation (B.1). The relations analogous 
to the scalar case are now

$$
\begin{aligned}
& \tilde{X}^{a} \equiv S \operatorname{curl} X^{a}=X_{V} \tilde{Q}_{V}^{a}, \\
& \dot{\tilde{X}}^{\langle a\rangle}=S \operatorname{curl} \dot{\tilde{X}}^{\langle a\rangle}=\dot{X}_{V} \tilde{Q}_{V}^{a}, \\
& \ddot{\tilde{X}}^{\langle a\rangle}=S \operatorname{curl} \ddot{\tilde{X}}^{\langle a\rangle}=\ddot{X}_{V} \tilde{Q}_{V}^{a} .
\end{aligned}
$$

Thus, for fixed comoving wave number $\mathrm{k}, X$ and $X_{S}$ as well as $\tilde{X}^{a}$ and $X_{V}$ will obey identical equations. We like to stress that all relations above are valid within the limits of our two-parameter approximation scheme.

\section{References}

[1] Grasso D and Rubinstein H R (2001), Phys. Rep. 348 163; Widrow L M (2002), Rev. Mod. Phys. 74775

[2] Kronberg P P (1994) Rep. Prog. Phys. 57 325; Han J-L and Wielebinski R (2002), Chin. J. Astron. Astrophys. 2293

[3] Parker E N (1979), Cosmological Magnetic Fields (Clarendon, Oxford); Zeldovich Y B, Ruzmaikin A A and Sokoloff D D (1983), Magnetic Fields in Astrophysics (McGraw-Hill, New York); Kronberg P P (1994), Rep. Prog. Phys. 57 325; Beck R, Brandenburg A, Moss D, Shukurov A A and Sokoloff D D (1996), Annu. Rev. Astron. Astrophys. 34155

[4] Kulsrud R M (1990), in Galactic and Extragalactic Magnetic Fields Eds. Beck R, Kronberg P P and Wielebinski R (Reidel, Dordrecht)

[5] Davis A-C, Lilley M and Törnkvist O (1999), Phys. Rev. D 600231301

[6] Calzetta E A, Kandus A, Mazzitelli F D and Wagner C E M (1998), Phys. Rev. D 57 7139; Kandus A, Calzetta E A, Mazzitelli F D and Wagner C E M (2000), Phys. Lett. B 472 287; Bassett B A, Pollifrone G, Tsujikawa S and Viniegra F (2001), Phys. Rev. D 63103515

[7] Giovannini M and Shaposhnikov M (2000), Phys. Rev. D 62103512

[8] Bertolami O and Mota D F (1999), Phys. Lett. B 455 96; Mazumdar A and Sheikh-Jabbari M M (2001) Phys. Rev. Lett. 87011301

[9] Tsagas C G, Dunsby P K S and Marklund M (2003), Phys. Lett. B 56117

[10] Tsagas C G and Barrow J D (1997), Class. Quantum Grav. 142539

[11] Tsagas C G and Maartens R (2000), Phys. Rev. D 61083519

[12] Hobbs S and Dunsby P K S (2000) Phys. Rev. D 62124007

[13] Harrison E R (1970), MNRAS 147 279; Harrison E R (1973), Phys. Rev. Lett. 3018

[14] Biermann J (1950) Z. Naturforsch. 5a 65

[15] Marklund M, Dunsby P K S, Servin M, Betschart G and Tsagas C G, (2003), Class. Quantum Grav. 201823

[16] Maartens R, Ellis G F R and Stoeger W (1996), Astron. Astrophys. 309 L7

[17] De Bernardis P et al (2000), Nature 404 955; Bennett C L et al (2003) ApJS 1481

[18] Maartens R (1997), Phys. Rev. D 55463

[19] Dunsby P K S, Bruni M and Ellis G F R (1992), ApJ 39557

[20] Harrison E R (1967), Rev. Mod. Phys. 39862 